\title{
Knowledge about Obstetric Danger Signs and Associated Factors among Antenatal Care Attendants
}

\author{
Tadele Girum¹, Mulugeta Shegaze ${ }^{2}$ \\ ${ }^{1}$ Department of Public health, College of Medicine and Health Sciences, Wolkite University, Wolkite, Ethiopia \\ ${ }^{2}$ Department of Public health, College of Medicine and Health Sciences, Arba Minch University, Arba Minch, Ethiopia
}

\begin{tabular}{l}
\hline Article Info \\
\hline Article history: \\
Received Oct 20, 2016 \\
Revised Dec 21, 2017 \\
Accepted Feb, 2017 \\
\hline
\end{tabular}

\section{Keyword:}

Knowledge

Danger Sign

Antenatal Care

\begin{abstract}
Knowledge of mothers about obstetric danger sign is the basic influencing factor for utilization of skilled maternal and neonatal cares which reduce maternal and new born mortality and morbidity. Therefore this study intended to assess knowledge about obstetric danger sign and identify associated factors among antenatal care attendants. Institutional based crosssectional study was conducted from April to June 2015 among 358 randomly selected antenatal care attendants. Epi Info version 7 and SPSS version 20 software were used for data entry and analysis respectively. Logistic regression was run to look for the association between dependent and explanatory variables; and using variables which have $p$-value $\leq 0.25$ binary logistic regression was fitted. Association presented in Odds ratio with 95\% confidence interval and significance determined at P-value less than 0.05. Most of the respondents able to name some of the accepted danger signs. Two hundred eight $(58 \%), 182(51 \%)$ and, $161(45 \%)$ of mother were knowledgeable for obstetric danger sign which could occur during pregnancy, at child birth and post-partum period respectively. The most mentioned dander signs were bleeding, retained placenta and prolonged labor. The factors that associated with Knowledge of obstetric danger signs were urban residence (AOR=2.6; 95\% CI: 1.8, 4.2), being literate $(\mathrm{AOR}=2.54 ; 95 \% \mathrm{CI}: 1.14,5.76)$, multiparity (AOR 1.5; 95\% CI: 1.14, 2.3) and being informed (AOR=3; 95\% CI: $1.6,6.4)$. The study showed that attendant's knowledge was low. This could be averted through strategies designed to address women's through health extension workers and health professionals.
\end{abstract}

Copyright @ 2017 Institute of Advanced Engineering and Science. All rights reserved.

\section{Corresponding Author:}

Tadele Girum,

Department of Public health,

College of Medicine and Health Sciences, Wolkite University,

Wolkite City, Ethiopia.

Email: girumtadele@yahoo.com

\section{INTRODUCTION}

Antenatal care (ANC) is a basic entry point for pregnant women to receive a broad range of promotive, preventive and curative health care services for the mother and her fetus. It also creates an opportunity to advice the women and her families about birth preparedness, potential obstetric complications and promotes the benefit of skilled attendance at birth and to encourage women to seek care for subroutine complains which could be of danger signs that have fatal outcomes [1]-[3].

Worldwide every day, the live of nearly 800 women is lost from preventable causes of adverse pregnancy outcomes. In 2013, 289000 women died during and following pregnancy and child birth. Almost all of these deaths occurred in low- resource settings, particularly in rural dwellers and among poor. The situation is serious in Sub-Sahara Africa, where 1 of every 16 women dies of pregnancy related causes during 
her lifetime, compared with only 1 in 2,800 women in developed regions. This big difference could reflect inequities in access to health services, and highlights the gap between the courtiers and among rural residents. Ethiopia, as one of the sub-Sahara African country; has the highest maternal morbidity mortality and associated complication [4]-[7].

Morbidities secondary to obstetric complication is by far higher than the cases of mortality, often leading to grave consequences [8],[9]. Obstetric complications are unpredictable; every pregnant woman may face the risk of sudden complications that could end in death or injury to herself or to her infant. Hence, it is necessary to design strategies to overcome such problems through appropriate health care system. Maternal morbidity and mortality could be prevented significantly if women and their families recognize obstetric danger signs and promptly seek health care. The commonest danger signs during pregnancy include severe vaginal bleeding, swollen hands/face and blurred vision. Key danger signs during labor and childbirth include severe vaginal bleeding, pro-longed labor, convulsions, and retained placenta [1],[10]-[12].

Since, these life threatening complications are treatable, and most of these deaths are avoidable if women with the complications are able to identify and seek appropriate emergency obstetric care which makes a difference between life and death awaring a mother and promoting her to seek care while she have any of the symptoms is very important. Lack of knowledge of the significance of symptoms of obstetric complications is one of the reasons of failure to identify and seek appropriate emergency care. Therefore, assessment of women's knowledge of obstetric danger signs and associated factors contributes for the planners and implementers to improve maternal health.

\section{MATERIALS AND METHODS}

\subsection{Study design and settings}

This cross-sectional study was conducted in Dilla university referral hospital, Southern Ethiopia through interviewing antenatal care attendants. DURH is the only referral hospital in Gedeo Zone providing care for 1,105,813 catchment population. There are two public health center and one referral hospital which offer ANC service and other maternal health packages. A large proportion of mothers are attending at the referral hospital.

\subsection{Study population and sampling technique}

All pregnant women who attended ANC service in Dilla university referral hospital were source populations. The sample size was calculated using single population proportion formula based on the following assumptions: Proportion of knowledge of danger sing during pregnancy $=30.4 \%$ [8], Significant level at $\alpha=0.05$, at $95 \%$ confidence interval, Margin of error is $5 \%$.and $10 \%$ nonresponse rate, the minimum sample size became 358 pregnant women. A systematic random sampling technique had been used to select study participants from pregnant mothers attending ANC in Dilla University Referral Hospital during the time of data collection.

\subsection{Data Collection Procedure and Data Quality Control}

After necessary modification and changes that had been made based on the pre - test the final survey was conducted by trained three midwifes by using structured checklist that developed from reviewing similar studies and the WHO antenatal cares follow up chart. The check list sought information on: sociodemographic characteristics, obstetric history, past experiences and physical findings.

\subsection{Study variables and Data analysis}

The Dependent variables is Knowledge on obstetric danger sign of pregnancy, labour and postpartum period; if she can mention at least two obstetric danger sign she was considered as knowledgeable and coded zero and other wise as not knowledgeable; and the independent variables were characteristics, obstetric history, past experiences and physical findings. After data collection, each questionnaire was checked for completeness and consistency. Then, Data was cleaned, coded and entered into Epi-info version 7 and exported to SPSS version 20 for analysis.

Exploratory data analysis carried out to check the levels of missing values, presence of influential outliers, independence of errors, multi-collinearity and normality. Binary and multiple logistic regressions were run to assess the association of various factors with late presentation. The final binary logistic regression model was fitted with variables significant at level of p.value $<0.25$. The fitness of the model was checked by Hosmer and Lemeshow test. The strength of association of predictor variables were assessed using odds ratio and significance of variables were reported by using $95 \%$ confidence interval and $\mathrm{p}$-values $<0.05$. 


\subsection{Ethical statement}

Ethical clearance and support letter was obtained from institutional review office of Dilla University. Permission to conduct the study also was obtained from Hospital Director office and department of Obstetrics and Gynecology. Informed consent was obtained from each study participants. Each respondents were informed about the purpose of the study and also that all data obtained from them were kept confidential by using codes instead of any personal identifiers.

\section{RESULTS AND DISCUSSION}

\subsection{Basic information of study participants}

Over all 358 antenatal care service attendants were interviewed at Dilla university referral hospital and none of them have refused to respond for the interview. The respondents' age ranged from 15 to 49 years with a mean age of 31.3 years; More than two third of the respondents were in the age group 20-34 years and 42 (11.7.) were attended grade 12 and above in their educational level. 199(55.6\%) of the respondents were Gedeo, 127 (35\%) were orthodox Christian by religion and 88.3\% were married. The majority $192(53.6 \%)$ of mothers were housewife and urban dwellers as shown in Table 1.

Table 1. Basic Information of The Respondents

\begin{tabular}{llcc}
\hline \multirow{4}{*}{ Residence } & Characteristics & $\mathrm{N}$ & percent \\
Religion & Urban & 293 & 82 \\
& Rural & 65 & 18 \\
& Orthodox & 127 & 35.5 \\
& Muslim & 51 & 14.2 \\
\multirow{5}{*}{ Marital status } & Protestant & 159 & 44.4 \\
& Other & 21 & 5.9 \\
Education & Married & 316 & 88.3 \\
& Not in marital union & 42 & 11.7 \\
& no education & 127 & 35.5 \\
& Primary & 73 & 20.4 \\
Occupation & secondary & 116 & 32.4 \\
& > 12 & 42 & 11.7 \\
& Private & 73 & 20.4 \\
& Civil servant & 42 & 11.7 \\
& Student & 51 & 14.2 \\
Ethnic group & Housewife & 192 & 53.6 \\
& Gedeo & 199 & 55.6 \\
& Oromo & 42 & 11.7 \\
& Sidama & 44 & 12.3 \\
& Others & 73 & 20.4 \\
\hline
\end{tabular}

\subsection{Obstetric characteristics of the attendants}

Almost half, 172(48.1\%) of the women were pregnant before the age of 20 years with mean age at first pregnancy being $20(3.7 \%)$. Of all the respondents $35(9.7 \%)$ women had five or more children with mean of 3.1 (SD 2.1) deliveries. Majority of the women (79.4\%) reported that their last pregnancy has been planned, and $45.4 \%$ were at home deliveries. From the total of mothers who had pregnancy before $15.4 \%$ had pregnancy associated complications; for that reason $74 \%$ went to health facility. The mean gestational age of the pregnant women during the interview time was 24 week. Majorities (57\%) had one and more ANC visits at time of data collection. Respondents were also been asked whether a health provider advised them on danger signs of pregnancy or not; $65.9 \%$ replies as they were informed about obstetrics danger signs and the possible measures.

\subsection{Knowledge about obstetric danger signs}

About $(87 \%)$ of mothers were able to spontaneously mentioned at least one obstetric danger sign which could occur at pregnancy and the most common mentioned danger signs were vaginal bleeding by 281 $(78.43 \%)$, Severe headache by $211(59.07 \%)$ and Swollen hands/face by $181(50.49 \%)$ of attendants. Two hundred eight $(58 \%)$ of women mentioned at least two danger signs during pregnancy. However forty seven $(13.0 \%)$ of mothers didn't know any danger signs of pregnancy as shown in Table 2.

Similarly, 294(82\%) of mothers mentioned at least one obstetric danger sign which could occur at labour and delivery mostly excessive bleeding by 409 (55\%), labor lasting more than 12 hours by 197(55) and placenta not delivered within 30 minutes after delivery of baby by 190(53\%) of the respondents respectively. One hundred eighty two $(51 \%)$ of respondents mentioned at least two danger signs during labour. Accordingly, 304 (85\%) of mothers mentioned at least one obstetric danger signs of post-partum 
period commonly mentioned include severe bleeding by 301(84.15), Severe weakness by $180(50.49)$ and Severe headache by 157(43.78) of the respondents. One hundred sixty one 161(45\%) of the pregnant women mentioned at least two serious danger signs during post-partum period as shown in Table 2 .

Generally, 208(58\%), 182(51\%) and, 161(45\%) of mother were knowledgeable for obstetric danger sign which could occur during pregnancy, at child birth and post-partum period respectively. The most mentioned dander signs were bleeding, retained placenta and prolonged labor; and the source of information was health professionals for $32 \%$ and Health extension workers for $26.9 \%$ of mothers.

Table 2. Knowledge about Obstetric Danger Sign of The Respondents

\begin{tabular}{lccc}
\multicolumn{1}{c}{ Danger signs } & \multicolumn{2}{c}{ Awareness of obstetric danger signs during } \\
& Pregnancy N(\%) & Intra-partum N $(\%)$ & Postpartum N(\%) \\
\hline Vaginal bleeding & $281(78.43)$ & $233(65)$ & $301(84.15)$ \\
Severe headache & $211(59.07)$ & $143(40)$ & $157(43.78)$ \\
Blurring of vision & $54(15.18)$ & $64(17.8)$ & $22(6.16)$ \\
High grade fever(sepsis) & $73(20.57)$ & $132(37)$ & $112(31.46)$ \\
Loss of consciousness & $73(20.57)$ & $114(32)$ & $136(38.17)$ \\
Convulsion & $40(11.11)$ & $\mathrm{NA}$ & $40(11.22)$ \\
Swollen hands/face & $181(50.49)$ & $\mathrm{NA}$ & $112(31.46)$ \\
Difficulty in breathing & $25(6.93)$ & $\mathrm{NA}$ & $34(9.57)$ \\
Severe weakness & $175(48.84)$ & $\mathrm{NA}$ & $180(50.49)$ \\
Severe abdominal pain & $68(18.92)$ & $\mathrm{NA}$ & $\mathrm{NA}$ \\
Decreased fetal movement & $69(19.25)$ & $\mathrm{NA}$ & $\mathrm{NA}$ \\
Rupture of membrane & $95(26.73)$ & $\mathrm{NA}$ & $\mathrm{NA}$ \\
Malodorous vaginal disch. & $\mathrm{NA}$ & $\mathrm{NA}$ & $95(26.5)$ \\
Labour $>12$ hours & $\mathrm{NA}$ & $197(55)$ & $\mathrm{NA}$ \\
Retained placenta & $\mathrm{NA}$ & $190(53)$ & $\mathrm{NA}$ \\
Others & $13(3.7)$ & $11(3)$ & $19(5.2)$ \\
\hline \multirow{2}{*}{ NA Not Assessed } & & & \\
\hline
\end{tabular}

$* \mathrm{NA}=$ Not Assessed

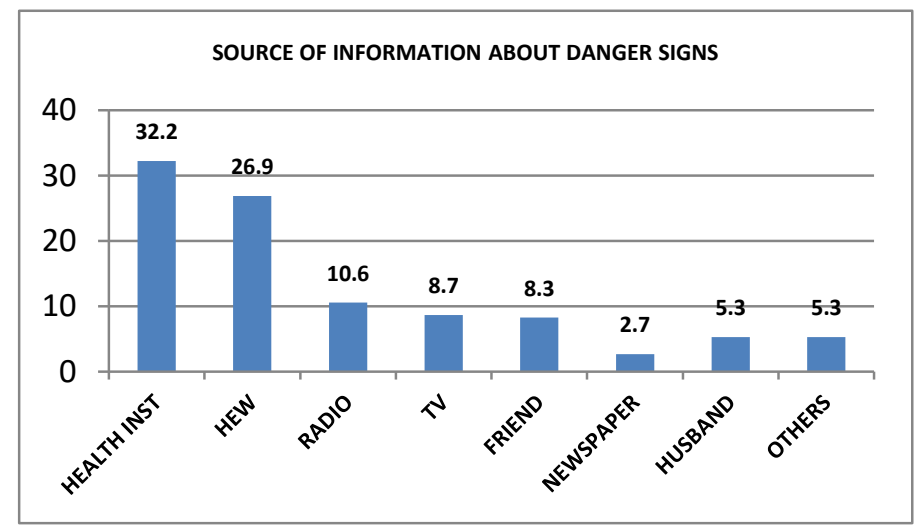

Figure 1. Source of Information about Obstetric Danger Signs

\subsection{Factors associated with knowledge of obstetric danger signs}

The effects of each independent variable on the knowledge of obstetric danger signs were tested using binary logistic regression and multivariable model was built by using variables having P-values less than 0.25 in bivariate analysis with forward step wise method. After controlling the effect of other variables residence, educational status and information provided an ANC were significantly associated with Knowledge of mothers on obstetric danger signs during pregnancy.

As compared to a woman living in rural area, a woman living in urban area was about three times more likely to know danger signs occurring pregnancy $(\mathrm{AOR}=3.2$ and $95 \% \mathrm{CI}=1.4,5.1)$. Mothers who can read and write were also $2.56(1.24,5.25)$ times more likely to know obstetric danger signs of pregnancy than illiterates and informed mothers were five times knowledgeable than their counter parts. The variables that associated with Knowledge of danger signs during labor were urban residence (AOR=2.6; 95\% CI: 1.8 , 4.2), being literate $(\mathrm{AOR}=2.54 ; 95 \% \mathrm{CI}: 1.14,5.76)$, multiparity (AOR 1.5; 95\% CI: 1.14, 2.3) and being informed $(\mathrm{AOR}=3 ; 95 \% \mathrm{CI}: 1.6,6.4)$. Accordingly the variables that were independently associated with knowledge about danger signs of the post natal period were urban residence (AOR=2.8; 95\% CI: 1.84, 4.7), being literate $(\mathrm{AOR}=2.47 ; 95 \% \mathrm{CI}: 1.14-6)$ and being informed $(\mathrm{AOR}=1.8 ; 95 \% \mathrm{CI}: 1.1-3.7)$. 
Table 3. Factors Associated with Knowledge of Obstetric Danger Signs among ANC Attendants

\begin{tabular}{|c|c|c|c|}
\hline \multirow{2}{*}{ Characteristics } & \multicolumn{3}{|c|}{ AOR $(95 \% \mathrm{CI})$ for knowledge about obstetric danger signs } \\
\hline & during pregnancy & during labor & during postnatal period \\
\hline \multicolumn{4}{|l|}{ Residence } \\
\hline Urban & $3.2(1.4-5.1)^{*}$ & $2.6(1.8-4.2) *$ & $2.8(1.84-4.7) *$ \\
\hline Rural & 1 & 1 & 1 \\
\hline \multicolumn{4}{|l|}{ Marital status } \\
\hline Married & $0.7(0.3-1.8)$ & $1.6(0.7-3.9)$ & $2.07(0.81-4.7)$ \\
\hline Not Married & 1 & 1 & 1 \\
\hline \multicolumn{4}{|l|}{ Mother's education } \\
\hline Illiterate & 1 & 1 & 1 \\
\hline Literates & $2.56(1.24-5.25) *$ & $2.54(1.14-5.76) *$ & $2.47(1.14-6.06) *$ \\
\hline \multicolumn{4}{|l|}{ Occupation } \\
\hline Housewife & 1 & 1 & 1 \\
\hline Gov't. employee & $1.3(0.46-6.44)$ & $1.07(0.36-4.05)$ & $2.1(0.76-3.15)$ \\
\hline Private & $1.1(0.49-4.44)$ & $1.17(0.55-4.85)$ & $1.31(0.63-3.48)$ \\
\hline \multicolumn{4}{|l|}{ Parity } \\
\hline Nulliparous & 1 & 1 & 1 \\
\hline Multiparity & $1.1(0.51-2.30)$ & $1.5(1.14-2.3) *$ & $0.9(0.28-1.42)$ \\
\hline \multicolumn{4}{|l|}{ Place of delivery } \\
\hline Home & 1 & 1 & 1 \\
\hline Institution & $0.91(0.41-1.32)$ & $1.09(0.33-1.85)$ & $0.99(0.55-1.97)$ \\
\hline \multicolumn{4}{|l|}{ Informed about ODS } \\
\hline Yes & $5.01(2.07-8.06)^{*}$ & $3(1.6-6.4) *$ & $1.8(1.1-3.70) *$ \\
\hline No & 1 & 1 & 1 \\
\hline
\end{tabular}

\subsection{Discussion}

The This study assessed the knowledge of ANC attendants on obstetric danger signs which occur during pregnancy, child birth and post-partum period and identified associated factors at Dilla university referral hospital which could help in identifying the existing situation, designing appropriate interventions and used as baseline for further studies in other part of the country.

In this study more than half $(58 \%)$ of mothers were knowledgeable about danger sign during pregnancy and Out of women under the study, $182(51 \%)$ were knowledgeable about danger signs during labour. Additionally, 161(45\%) were knowledgeable about danger signs during postpartum period which is much higher than studies conducted in Aleta Wondo and Goba district (Ethiopia) in which only 30.4 \& $31.9 \%$.of women were knowledgeable for danger signs during pregnancy, only $41 \%$ \& 27 of women were knowledgeable for danger signs during pregnancy and only $37.7 \%$ \&22.1\% of women were knowledgeable for post-partum period [14]. It is also higher than the studies conducted in in rural Tanzania and Uganda [15],[16].

The difference could be due to difference in socio-demography of the participants, the time difference and measurement difference. In Goba knowledge was assessed by mentioning 3 danger signs which could create difference, the study of Aleta wondo was conducted 8 years back after which a lot of health promotion activities were implemented and moreover the participants of this study were mainly of urban dwellers which could have better knowledge.

Among the obstetric danger signs, Vaginal bleeding was the most recognized obstetric danger sign during pregnancy, $281(78.43 \%)$, labour, 233(65\%) and postpartum period, 301(84.15\%). It is in line with other similar studies [12]-[15]. Retained placenta, 190(53\%) and labour lasting greater than 12 hours, $197(55 \%)$ were the two major obstetric danger signs mentioned by the respondents during child birth. These findings were also in line with the study conducted in Aleta Wondo where knowledge of retained placenta was (51.4\%) and labour lasting greater than 12 hours was (43.2\%) [13]. However the proportion of mothers mentioning each sign were higher in our study than others.

According to this study urban dweller attendants were 3.2 times more likely to mention at least two danger signs during pregnancy, 2.6 times more likely to mention danger signs during labour and 2.8 times complications of post-partum period than their counterparts of rural women. This variable was also found to have a significant association with knowledge of obstetrics danger signs during pregnancy, child birth and postpartum period in other study [14],[15]. This could be due to the fact that urban residents have better access to health information and maternal health services as compared with rural counterparts.

The odds of Literacy to have knowledge of obstetric danger signs were higher than illiterates. Literates were 2.56, 2.54 and 2.47 times more likely to be knowledgeable on obstetric danger signs of pregnancy, labour and post-partum periods respectively. It is obvious that the basic knowledge of mothers are important for self-care as well, educated mothers are more likely to read about and follow information dissimilated and are aware to seek health care; which could help them to acquire knowledge on obstetric 
danger signs. Association of education with knowledge of obstetric danger sigh was also been reported from other studies [15],[16].

As compared to nulliparous, multiparous were about 1.5 times more likely to mention at least two danger signs during labour. This could be explained by the fact that, a woman who has previous child birth experience, recognize more from her exposure and she is also more likely to seek health care and receive health education. The other most important predictor of women's knowledge of obstetric danger sign was information provided about danger signs. Those mothers who received information from health extension workers and health professionals were 5.01, 3 and 1.8 times more likely to be knowledgeable about obstetrics complications of pregnancy, labour and post-partum respectively than their counterparts. Also in the other studies information provision was associated with knowledge during pregnancy. However it was failed to show association with knowledge during labour and post-partum period [13].

\section{CONCLUSION AND RECOMMENDATION}

This study showed that the level of knowledge was low; even thou it is higher than other similar studies which indicate the large proportions of pregnant women who do not have the knowledge are likely to delay in deciding to seek care and further results in maternal and child death. The major factors affecting mother's knowledge were their residence, educational status and information received by health extension workers or health professionals. Therefore, the health professionals should design appropriate strategies including provision of targeted health education or provide information, education and communication to pregnant mothers to increase their awareness and thus enable early recognition of serious health problems during pregnancy, labor and post-partal period.

\section{ACKNOWLEDGEMENTS}

I would like to sincerely thank the study participants.

\section{REFERENCES}

[1] Wafaa R. and Rasha E., "Women's Awareness of Danger Signs of Obstetrics Complications," Journal of American Science, vol/issue: 6(10), 2010.

[2] JHPIEGO, "Maternal and Neonatal Health Pro-gram. Birth Preparedness and Complication Readiness: A Matrix of Shared Responsibilities," Maryland, USA, JHPIEGO, 2004.

[3] WHO, "Standards for Maternal and Neonatal Care: Birth and emergency preparedness in antenatal care: Department of Making Pregnancy Safer (MPS)," Geneva, World Health Organization, 2006.

[4] Federal Ministry of Health (FMOH), Ethiopia. "Health and Health Related Indicators: 2006/7," Addis Ababa, Ethiopia, FMOH, 2007.

[5] Federal Ministry of Health (FMOH), Ethiopia, "Health and Health Related Indicators: 2006/7," Addis Ababa, Ethiopia, FMOH, 2008.

[6] WHO, "Mother-Baby Package," Geneva, 2010.

[7] WHO, UNICEF, UNFPA, WORLD Bank, "Maternal mortality," Geneva, 2012.

[8] World Health Organization (WHO), "Pregnancy is special let's make it safe: World Health Day, Safe Motherhood," Geneva, Switzerland, WHO, 1998.

[9] Hill K., et al., "Estimates of maternal mortality worldwide between 1990 and 2005," Lancet, vol. 370, pp. 1311-19, 2007.

[10] Mihret H. and Mesganaw F., "Birth Preparedness and Complication Readiness among women in Adigrat town, north Ethiopia," Ethiop J Health Dev., vol/issue: 22(1), 2008.

[11] Hiluf M. and Fantahun M., "Birth Preparedness and Complication Readiness among women in Adigrat town, north Ethiopia," Ethiop J Health Dev., vol/issue: 22(1), pp. 14-20, 2007.

[12] Ekabua J., et al., "Awareness of Birth Preparedness and Complication Readiness in Southeastern Nigeria," $S R N$ Obstetrics and Gynecology, vol. 6, 2011.

[13] Mesay H., et al., "Knowledge about obstetric danger signs among pregnant women in Aleta Wondo district, Sidama Zone, Southern Ethiopia," Ethiop J Health Sci., vol/issue: 20(1), pp. 25 - 32, 2010.

[14] Daniel B. and Desalegn M., "Knowledge of obstetric danger signs among child bearing age women in Goba district, Ethiopia: a cross-sectional study," BMC Pregnancy and Childbirth, vol. 15, pp. 77, 2015.

[15] Pembe A. B., et al., "Rural Tanzanian women's awareness of danger signs of obstetriccomplications," BMC Pregnancy Childbirth, vol/issue: 9(12), 2009.

[16] Kabakyenga J. K., et al., "Petterss on KO: Knowledge of obstetric danger signs and birth preparedness among women in rural Uganda," Reprod Health, vol/issue: 8(33), 2011. 